\title{
Validez convergente de la escala POMS-VIC Validation and convergent analysis of the scale POMS-VIC
}

\author{
*Pablo José Borges, **Roberto Ruiz-Barquín, **Ricardo De la Vega
}

*Universidad de La Laguna (España), **Universidad Autónoma de Madrid (España)

\begin{abstract}
Resumen. Este estudio pretende analizar la validez convergente de un instrumento para la medición tridimensional de los estados de ánimo: el POMS-VIC. El instrumento fue aplicado a 62 estudiantes del Servicio de deportes de la UAM. Se compararon las puntuaciones ofrecidas por los deportistas al completar el POMS-VIC y otras pruebas similares (PIED) Perfil Interactivo de Estados de ánimo (Barrios \& González, 2012); (STAI) State-Trait Anxiety Inventory (Guillén-Riquelme \& Buela-Casal, 2011) y el (CSAI-2R) Revised Competitive State Anxiety Inventory-2 (Andrade et al., 2007). Se calculó el coeficiente de correlación por rangos de Spearman y contraste no paramétrico de grupos mediante la prueba U de Mann Whitney. Apreciando correlaciones medias entre PIED y POMS_Intensidad (rho=-.61),POMS_Valencia (rho=.34) y POMS_Control $($ rho $=.43)$. Así como entre POMS_Intensidad $y$ las tres subescalas del CSAI (Cognitivo, $r h o=-.31)$, (Somático, rho $=-.55)$ y (Autoconfianza, $r h o=.56$ ). Se concluye que existe una relación significativa y relevante entre el POMS-VIC y los diferentes instrumentos considerados. Este resultado indica que la propuesta es válida para la medida del estado emocional y de los estados de ánimo, pudiéndose utilizar este instrumento en el ámbito deportivo. Por tanto, se considera que el instrumento puede tener una repercusión práctica y significativa en el ámbito de la psicología del deporte.
\end{abstract}

Palabras clave: POMS-VIC, estado emocional, validación, análisis convergente.

\begin{abstract}
This study aims to analyze the convergent validity of an instrument for the three-dimensional measurement of mood states: the POMS-VIC. The instrument was applied to 62 students from the UAM Sports Service. The scores offered by the athletes when completing the POMS-VIC and other similar tests (PIED) Interactive Profile of Moods, (Barrios \& González, 2012); (STAI) State-Trait Anxiety Inventory (Guillén-Riquelme \& Buela-Casal, 2011) and (CSAI-2R) Revised Competitive State Anxiety Inventory-2 (Andrade et al., 2007) were compared. The correlation coefficient was calculated by Spearman rank and non-parametric group contrast using the Mann Whitney U test. Appreciating mean correlations between PIED and POMS_Intensidad (rho=-.61), POMS_Valencia $(r h o=.34)$ and POMS_Control ( $r h o=.43)$. As well as between POMS_Intensidad and the three subscales of CSAI (Cognitive, rho=-.31), (Somatic, rho $=-.55)$ and (Selfconfidence, rho $=.56$ ). It is concluded that there is a considerable relationship between the POMS-VIC and the considered instruments. This result implies that the proposal scale is valid for the mood state measurement and allows its use in the sports field. Therefore, the instrument is considered to have an important practical impact for sport psychology.
\end{abstract}

Key words: POMS-VIC, emotional state, validation, convergent analysis.

\section{Introducción}

¿A qué se hace referencia cuando se habla del término emoción?. Al respecto, existe una considerable diversidad de opiniones, lo que ha provocado que se hayan producido diferentes aproximaciones a su estudio desde diferentes ramas de conocimiento, como la psicología, sociología, filosofía, neurociencia y recientemente se ha introducido en el ámbito deportivo (Puig, 2012).

En psicología, las emociones constituyen una de las parcelas más atractivas, pero han inspirado al mismo tiempo una de sus páginas más caóticas, debido a la diferenciación y conceptualización de los diferentes constructos (sentimiento, afecto, emoción y estado de

Fecha recepción: 25-01-21. Fecha de aceptación: 02-06-21

Pablo José Borges

pborher@gmail.com ánimo) cuyos límites en ocasiones se entremezclan. Existiendo en la actualidad múltiples aproximaciones teóricas que mantienen puntos de vista diferentes. En este sentido, en este trabajo se parte de la diferenciación conceptual señalada en De la Vega, Galán, Ruiz y Tejero (2013), en donde los dos ejes que marcarían las diferencias fundamentales entre los términos señalados serían la duración temporal de la vivencia emocional y su relación con los procesos cognitivos (Cerin, Szabo, Hunt \& Williams, 2000), así como el nivel de estabilidad o de fluctuación que tienen a través de las situaciones deportivas.

La conceptualización del estado de ánimo implica analizarlo como un constructo amplio que se refiere al estado permanente en el que un individuo se encuentra, produciéndose de forma gradual. Por esta naturaleza gradual del estado de ánimo, se puede diferenciar de la emoción en su menor nivel de intensidad aguda ante 
la aparición de un estímulos específico, lo que suele implicar la existencia de creencias sobre la posibilidad de experimentar afecto positivo o negativo en el futuro que poseen un potente matiz explicativo del estado de ánimo vivenciado (De la Vega et al., 2014).

Esto ha llevado a Hanin (2007) a señalar que se ha subestimado o ignorado la influencia de las emociones en la psicología deportiva. En parte, este hecho se debe a que tradicionalmente se ha medido el estado emocional únicamente considerando la medida de la intensidad de las sensaciones que configurarían el estado anímico del deportista. Lo cual, a juicio de los autores, es una medida no del todo completa, aunque importante, para analizar de una manera global, la influencia del estado de ánimo en el rendimiento deportivo. Motivo por el cual se presenta la escala POMS-VIC, que parte del modelo tridimensional de la emoción propuesto por Peter Lang (2010), y que ubica al deportista en tres ejes: Intensidad, Valencia y Control (De la Vega et al., 2014). Desde esta perspectiva la respuesta emocional de la persona presenta diferentes dimensiones no solo determinadas por la intensidad. Es por ello, que la innovación que pretende el presente trabajo es la inclusión de diferentes parámetros de la respuesta emocional no solo basados en el parámetro de intensidad.

Y es que en relación al área del deporte y el ejercicio, las emociones y, en particular, los estados de ánimo, han constituido un contenido esencial de la evaluación psicológica del entrenamiento y el rendimiento deportivo, por la incidencia en la manera de percibir y enfrentar las demandas que se le presentan al deportista durante su preparación y la competición. En este sentido, Andrade, Arce y Seoane (2000), exponen que la escala para la medida del Perfil de los Estados de Ánimo (POMS) diseñada por McNair, Lorr y Dropelmann (1971), concebida originalmente en el ámbito clínico, ha sido la más utilizada en el deporte. Este instrumento presenta una importante capacidad predictiva en el desarrollo de predicciones acerca del rendimiento de los deportistas a partir de las características de sus estados de ánimo (Andrade et al., 2000). A pesar de ello, este instrumento también presenta algunas limitaciones. Debido a estas limitaciones, surge esta nueva propuesta de evaluación derivada del POMS original (Morgan, 1980), evaluando los estados de ánimo desde una perspectiva multidimensional. En el presente estudio se desarrollan diferentes análisis que pretenden validar el instrumento estableciéndose relaciones con otros instrumentos psicológicos ya validados y ampliamente utilizados en el ámbito de investigación y práctico de la
Psicología del Deporte. Se pretende ofrecer una visión más completa sobre los niveles de respuesta emocional del deportista ante determinadas situaciones deportivas, evaluando tres dimensiones: la evaluación tradicional de la dimensión Intensidad, y la incorporación a la escala de dos parámetros más como son Valencia y Control (De la Vega et al., 2014). Esta perspectiva tridimensional podría permitir una evaluación holística y global de los estados emocionales del deportista, intentando reducir una información relevante del estado emocional del deportista no solo respondiendo al parámetro intensidad.

En esta línea, existen diferentes trabajos que han obtenido relaciones relevantes entre la percepción de los estados de ánimo y un aumento en el rendimiento. Ya sea desde el ámbito laboral (Ben-Zur \& Yagil, 2005), académico (Bar-On \& Parker, 2000, Fierro-Suero, Velázquez-Ahumada \& Fernández-Espínola, 2021; Molano-Tobar, Rojas \& Vélez, 2021) o deportivo (Arroyo, González \& Arruza, 2020). En este ámbito, autores como Andrade et al. (2000) citan al menos 257 estudios en los que se ha aplicado el POMS a practicantes 32 modalidades deportivas diferentes, y donde empieza a cobrar relevancia la inclusión de planes de entrenamiento psicológicos.

En este sentido, y considerando que uno de los objetivos de la Psicología del Deporte es dotar al deportista de recursos para hacer frente a demandas psicológicas derivadas de la competición y el entrenamiento a fin de que consiga aumentar su rendimiento y disfrutar más de su actividad deportiva, es determinante el análisis de la influencia y las relaciones entre el estado de ánimo precompetitivo y el rendimiento deportivo. Esta importancia no solo tiene implicaciones en el deporte de alto rendimiento o profesional, sino también en etapas o procesos de formación deportiva (Abenza et al., 2014).

Es por ello, que dado el gran número de investigaciones, en las cuales el POMS ha sido aplicado a deportistas, era inevitable la aparición de una pluralidad de resultados, que no ha estado exenta de críticas (Prapavessis, 2000). Algunas de estas críticas se relacionan con el empleo de metodologías diversas, la evaluación de sujetos con distinto nivel de entrenamiento y edad, o la evaluación del estado emocional en diferentes momentos de la temporada o competición.

Según la revisión realizada en la literatura científica, la principal limitación que se ha encontrado la literatura ha sido considerar el estado emocional desde un modelo unidimensional, valorando únicamente la inten- 
sidad con que el deportista vive una emoción y no prestando atención a la valoración que hace sobre la misma y el grado de control que ejerce sobre ella. Justificando así la creación de esta nueva escala que integra estas tres esferas (Intensidad, Valencia y Control).

Por este motivo, este trabajo pretende comprobar la validez convergente de la escala POMS-VIC, mediante la aplicación de la misma, y las pruebas Revised Competitive State Anxiety Inventory-2 (CSAI-2R) (Andrade et al., 2007; adaptado de Martens et al., 1990), State-Trait Anxiety Inventory (STAI) (Guillén-Riquelme \& Buela-Casal, 2011; adaptado de Spielberger et al., 1970) y el Perfil Interactivo de Estados de ánimo (PIED) (Barrios \& González, 2012). Justificándose el uso de estos cuestionarios para la validación de la escala propuesta porque son ampliamente usados en Psicología del Deporte para la medida del estado de ánimo y presentan una estructura similar a la escala que se quiere validar.

Dadas las importantes diferencias psicológicas, de los procesos emocionales y del estado de ánimo en el ámbito de la práctica de actividad física y el ámbito deportivo en función de la edad y sexo de los practicantes y deportistas (Balaguer et al., 1993; Ruiz, 2005), el presente estudio analiza el instrumento POMS-VIC estableciendo posibles diferencias de sus respuestas en función de estos dos parámetros.

\section{Metodología}

\section{Participantes}

Para la realización de este estudio han participado 62 estudiantes universitarios del Servicio de Deportes, mayores de edad ( $M=30.85 ; D T=10.48$ años), de los cuales 38 fueron hombres y 24 mujeres. La muestra fue seleccionada mediante muestreo incidental por el único motivo de acceso viable. La participación fue voluntaria, anónima y consentida; y se respetó la Declaración de Helsinki en todos sus términos.

\section{Instrumentos}

Para la realización de este estudio, se emplearon los cuestionarios CSAI-2R, propuesto por Martens et al., (1990); y adaptado al castellano por Andrade etal. (2007), está compuesto por 17 ítems, que se distribuye en tres subescalas: Ansiedad cognitiva, Ansiedad somática y Autoconfianza. El cuestionario STAI, (Guillén-Riquelme \& Buela-Casal, 2011; adaptado de Spielberger et al., 1970), se compone de veinte ítems para cada una de las subescalas (ansiedad estado y ansiedad rasgo). Mientras que el PIED, (Barrios \& González, 2012), está compuesto por seis listas que recogen, cada una, tres adjetivos correspondientes a cada factor del POMS clásico (Tensión, Depresión, Cólera, Vigor, Fatiga y Confusión), y el POMS-VIC (De la Vega et al., 2014), en su versión ordenada, compuesta por 33 ítems cada una de las tres escalas y medidas con un rango de respuesta tipo Likert de cinco puntos, usando esta versión del POMS porque se cumplen tres criterios señalados por los autores: ítems comprensibles, conceptualmente próximos al factor con el que se corresponden y con un mínimo de 4 ítems por factor, ofreciendo la siguiente estructura factorial: Tensión (4 ítems), Estado deprimido (9 ítems), Cólera (4 ítems), Vigor (4 ítems), Fatiga (4 ítems), Confusión (4 ítems) y Amistad (4 items). Todos ellos validados, ampliamente usados en el ámbito de la Psicología del Deporte y con una elevada fiabilidad interna, tal y como se recoge en la tabla 1 que muestra los valores alfa encontrados en la muestra analizada.

\begin{tabular}{|c|c|c|c|c|c|c|}
\hline \multirow[b]{2}{*}{ Variable } & \multicolumn{3}{|c|}{ POMS-VIC } & \multirow[t]{2}{*}{ CSAI } & \multirow[t]{2}{*}{ STAI } & \multirow[t]{2}{*}{ PIED } \\
\hline & Intensidad & Valencia & Control & & & \\
\hline Tensión & 0.88 & 0.95 & 0.94 & & & \\
\hline Depresión & 0.87 & 0.98 & 0.95 & & & \\
\hline Cólera & 0.89 & 0.98 & 0.96 & & & \\
\hline Vigor & 0.94 & 0.97 & 0.94 & & & \\
\hline Fatiga & 0.81 & 0.87 & 0.96 & & & \\
\hline Confusión & 0.77 & 0.95 & 0.91 & & & \\
\hline Amistad & 0.87 & 0.74 & 0.77 & & & \\
\hline Somática & & & & 0.80 & & \\
\hline Cognitiva & & & & 0.87 & & \\
\hline Autoconfianza & & & & 0.91 & & \\
\hline Escala & 0.74 & 0.97 & 0.96 & 0.76 & 0.70 & 0.71 \\
\hline
\end{tabular}

En la versión actual presentada se emplean tres escalas: la clásica de Intensidad del estado de ánimo, y dos nuevas, Valencia (agradable-desagradable), y Control (sin control sobre el estado de ánimo experimentado, o con mucho control sobre el estado de ánimo experimentado), siendo congruentes con el marco teórico de partida planteado en este trabajo.

\section{Procedimiento}

La investigación ha seguido un diseño instrumental (Montero \& León, 2007). Se contactó con diferentes profesores universitarios, con el fin de obtener la mayor muestra posible para la investigación. Todos los estudiantes que aceptaron formar parte del estudio, firmaron el consentimiento informado y fueron avisados de que completarían varios cuestionarios sobre el estado emocional (POMS-VIC, CSAI-2R, STAI y PIED, según este orden en única sesión y con una duración aproximada de 20 minutos) con el fin de validar la nueva escala propuesta. 


\section{Análisis de datos}

Con la intención de describir los niveles de los estados de ánimo se estimó de cada variable su media aritmética $(M)$, la desviación típica $(D T)$, se analizó la fiabilidad interna de las escalas y dimensiones mediante la prueba alfa de Crombach y se analizó la distribución de los datos (normalidad) mediante la prueba de ShapiroWilk. Para el establecimiento de los correspondientes análisis correlacionales y los análisis de diferencia de medias para dos muestras independientes, se aplicó el coeficiente de correlación rho de Spearman y la comparación de medias mediante el estadístico U de MannWhitney. Este estadístico se interpretó de acuerdo a Salkind (1999), considerando correlaciones muy bajas entre .00 y .20; bajas entre .21 y .40 ; moderadas entre .41 y .60 ; altas entre .61 y .80 y muy altas entre .81 y 1. Los análisis se realizaron mediante el paquete estadístico $\mathrm{R}$, estableciendo un nivel de confianza del 95\% $(p<.05)$.

\section{Resultados}

Con respecto a los análisis descriptivos, la tabla 2, muestra los valores encontrados en este estudio: media aritmética $(M)$ y desviación típica $(D T)$, relacionadas con las puntuaciones obtenidas en el POMS-VIC y los

Tabla 2 .

Estadísticos descriptivos POMS-VIC

\begin{tabular}{|c|c|c|c|c|c|c|c|c|c|c|c|c|}
\hline \multirow[b]{3}{*}{ Variable } & \multicolumn{11}{|c|}{ (a) Intensidad } & \multirow{3}{*}{$\begin{array}{c}\text { Género } \\
U(p) \\
\end{array}$} \\
\hline & \multicolumn{2}{|c|}{ Total } & \multicolumn{2}{|c|}{$\begin{array}{c}\text { Edad }<25 \\
(n=26)\end{array}$} & \multicolumn{2}{|c|}{$\begin{array}{c}\text { Edad }>25 \\
(n=36)\end{array}$} & \multirow{2}{*}{$\begin{array}{l}\text { Edad } \\
U(p)\end{array}$} & \multicolumn{2}{|c|}{$\begin{array}{c}\text { Hombre } \\
(n=38)\end{array}$} & \multicolumn{2}{|c|}{$\begin{array}{l}\text { Mujer } \\
(n=24)\end{array}$} & \\
\hline & $M$ & $D T$ & $M$ & $D T$ & $M$ & $D T$ & & $M$ & $D T$ & $M$ & $D T$ & \\
\hline Tensión & 1.06 & 0.98 & 0.97 & 0.93 & 1.05 & 0.93 & 0.68 & 1.12 & 1.08 & 0.97 & 0.82 & 0.86 \\
\hline Depresión & 0.52 & 0.67 & 0.44 & 0.73 & 0.53 & 0.57 & 0.12 & 0.49 & 0.66 & 0.56 & 0.69 & 0.27 \\
\hline Cólera & 0.42 & 0.76 & 0.38 & 0.82 & 0.38 & 0.63 & 0.62 & 0.39 & 0.72 & 0.46 & 0.84 & 0.82 \\
\hline Vigor & 2.55 & 0.93 & 2.45 & 0.88 & 2.66 & 0.94 & 0.48 & 2.62 & 0.99 & 2.44 & 0.82 & 0.30 \\
\hline Fatiga & 1.16 & 0.94 & 0.89 & 0.85 & 1.31 & 0.96 & 0.08 & 1.05 & 0.98 & 1.33 & 0.87 & 0.18 \\
\hline Confusión & 0.71 & 0.85 & 0.58 & 0.78 & 0.74 & 0.85 & 0.37 & 0.70 & 0.91 & 0.72 & 0.78 & 0.58 \\
\hline Amistad & 2.77 & 0.64 & 2.77 & 0.72 & 2.79 & 0.58 & 0.77 & 2.83 & 0.64 & 2.68 & 0.64 & 0.38 \\
\hline Escala & \multicolumn{2}{|c|}{103.2319 .491} & 105.68 & 19.76 & 103.14 & 17.13 & 0.40 & 104.32 & $220.491 \mathrm{C}$ & 101.50 & 18.09 & 0.30 \\
\hline \multicolumn{13}{|c|}{ (b) Valencia } \\
\hline & \multicolumn{2}{|c|}{ Total } & \multicolumn{2}{|c|}{$\begin{array}{c}\text { Edad }<25 \\
(\mathrm{n}=26)\end{array}$} & \multicolumn{2}{|c|}{$\begin{array}{c}\text { Edad }>25 \\
(n=36)\end{array}$} & Edad & \multicolumn{2}{|c|}{$\begin{array}{l}\text { Hombre } \\
(\mathrm{n}=38)\end{array}$} & \multicolumn{2}{|c|}{$\begin{array}{l}\text { Mujer } \\
(\mathrm{n}=24)\end{array}$} & \\
\hline Variable & $M$ & $D T$ & $M$ & $D T$ & $M$ & $D T$ & $U(p)$ & $M$ & $D T$ & $M$ & $D T$ & $U(p)$ \\
\hline Tensión & 1.90 & 1.10 & 1.75 & 1.17 & 2.02 & 1.05 & $0.04 *$ & 2.14 & 1.11 & 1.51 & 0.98 & 0.91 \\
\hline Depresión & 1.90 & 1.36 & 1.85 & 1.52 & 1.96 & 1.28 & 0.69 & 2.29 & 1.25 & 1.29 & 1.33 & $0.01 * *$ \\
\hline Cólera & 1.98 & 1.35 & 1.89 & 1.48 & 2.06 & 1.29 & 0.50 & 2.30 & 1.24 & 1.47 & 1.39 & $0.01 * *$ \\
\hline Vigor & 3.42 & 0.70 & 3.42 & 0.81 & 3.48 & 0.50 & 0.61 & 3.38 & 0.72 & 3.47 & 0.69 & 0.63 \\
\hline Fatiga & 1.79 & 0.92 & 1.75 & 0.99 & 1.84 & 0.89 & 0.66 & 2.05 & 0.98 & 1.39 & 0.65 & $0.01 * *$ \\
\hline Confusión & 1.90 & 1.16 & 2 & 1.23 & 1.85 & 1.13 & 0.79 & 2.26 & 1.20 & 1.32 & 0.83 & $0.00 * *$ \\
\hline Amistad & 3.16 & 0.55 & 3.22 & 0.54 & 3.15 & 0.53 & 0.53 & 3.21 & 0.56 & 3.07 & 0.55 & 0.28 \\
\hline Escala & \multicolumn{4}{|c|}{112.9227 .51114 .3231 .22} & 111.81 & 25.43 & 0.70 & 104.79 & 26.19 & 125.79 & 24.92 & 0.01 \\
\hline \multicolumn{13}{|c|}{ (c) Control } \\
\hline & \multicolumn{2}{|c|}{ Total } & \multicolumn{2}{|c|}{$\begin{array}{c}\text { Edad }<25 \\
(\mathrm{n}=26)\end{array}$} & \multicolumn{2}{|c|}{$\begin{array}{c}\text { Edad }>25 \\
\quad(n=36)\end{array}$} & Edad & \multicolumn{2}{|c|}{$\begin{array}{c}\text { Hombre } \\
(\mathrm{n}=38)\end{array}$} & \multicolumn{2}{|c|}{$\begin{array}{l}\text { Mujer } \\
(\mathrm{n}=24)\end{array}$} & Género \\
\hline Variable & $M$ & $D T$ & $M$ & DT & $M$ & $D T$ & $U(p)$ & $M$ & $D T$ & $M$ & $D T$ & $U(p)$ \\
\hline Tensión & 2.31 & 0.98 & 2.46 & 1.00 & 2.24 & 0.97 & 0.06 & 2.43 & 1.03 & 2.13 & $0.89 \mathrm{c}$ & $0.00 * * *$ \\
\hline Depresión & 2.33 & 1.03 & 2.43 & 1.18 & 2.28 & 0.93 & 0.59 & 2.60 & 1.01 & 1.90 & $0.92 \mathrm{c}$ & $0.00 * * *$ \\
\hline Cólera & 2.34 & 1.19 & 2.47 & 1.23 & 2.28 & 1.18 & 0.56 & 2.55 & 1.15 & 2 & 1.21 & 0.07 \\
\hline Vigor & 3.04 & 0.74 & 2.98 & 0.80 & 3.12 & 0.67 & 0.60 & 3.13 & 0.67 & 2.91 & 0.83 & 0.43 \\
\hline Fatiga & 2.11 & 0.99 & 2.23 & 1.17 & 2.06 & 0.85 & 0.66 & 2.24 & 1.11 & 1.91 & 0.74 & 0.38 \\
\hline Confusión & 2.27 & 1.08 & 2.48 & 1.07 & 2.16 & 1.07 & 0.26 & 2.45 & 1.19 & 1.98 & 0.83 & 0.14 \\
\hline Amistad & 3.10 & 0.61 & 3.08 & 0.59 & 3.16 & 0.60 & 0.61 & 3.21 & 0.53 & 2.94 & 0.70 & 0.08 \\
\hline Escala & 101.52 & 21.57 & 97.80 & 23.20 & 103.64 & 20.48 & 0.33 & 97.29 & 22.1 & 108.2 & 19.12 & 0.05 \\
\hline
\end{tabular}

grupos considerados. En esta tabla se puede apreciar puntuaciones elevadas si se comparan con resultados previos (De la Vega et al., 2014) para los factores considerados positivos (Vigor y Amistad) y puntuaciones bajas para los factores considerados negativos (Tensión, Depresión, Cólera, Fatiga y Confusión), considerando las variables, edad y sexo de los participantes. Asimismo, se aprecia significación estadística entre los factores Depresión, Cólera, Fatiga y Confusión $(p<.01)$ de la escala Valencia; Depresión y Tensión respecto a la escala Control $(p<.001)$ al comparar las puntuaciones considerando la variable sexo; y en el factor Tensión de la escala Valencia $(p=.04 ; p<.05)$ considerando la variable edad.

La tabla 3 ofrece los valores del instrumento POMSVIC al comparar los resultados de su aplicación a los participantes, con los cuestionarios administrados PIED, STAI y CSAI-2R, teniendo en cuenta sus tres variantes (CSAIcognitivo, CSAIsomático, y CSAIautoconfianza), mediante la prueba rho de Spearman.

\begin{tabular}{|c|c|c|c|c|c|c|}
\hline \multicolumn{7}{|c|}{ (a) Intensidad } \\
\hline \multicolumn{7}{|c|}{ Rho Spearman } \\
\hline Variable & Shapiro (p) & PIED & STAI & CSAI_C & CSAI_S & CSAI_A \\
\hline Tensión & 0.01 & $0.50 *$ & 0.07 & $0.40 *$ & $0.53 *$ & $-0.31 *$ \\
\hline Depresión & 0.00 & $0.59 *$ & 0.18 & $0.26 *$ & $0.41 *$ & $-0.53 *$ \\
\hline Cólera & 0.00 & $0.48 *$ & 0.07 & 0.22 & $0.36 *$ & -0.23 \\
\hline Vigor & 0.28 & -0.18 & $-0.32 *$ & -0.15 & $-0.27 *$ & $0.50 *$ \\
\hline Fatiga & 0.05 & $0.62 *$ & -0.15 & 0.16 & $0.44 *$ & $-0.35 *$ \\
\hline Confusión & 0.00 & $0.45 *$ & 0.17 & 0.22 & $0.37 *$ & $-0.39 *$ \\
\hline Amistad & 0.28 & -0.17 & $-0.33 *$ & -0.17 & $-0.25 *$ & $0.45 *$ \\
\hline Escala & 0.00 & $-0.61 *$ & -0.20 & $-0.31 *$ & $-0.55 *$ & $0.56 *$ \\
\hline \multicolumn{7}{|c|}{ (b) Valencia } \\
\hline \multicolumn{7}{|c|}{ Rho Spearman } \\
\hline Variable & Shapiro & PIED & STAI & CSAI_C & CSAI_S & CSAI_A \\
\hline Tensión & 0.00 & $-0.44 *$ & 0.04 & -0.21 & $-0.38^{*}$ & $0.32 *$ \\
\hline Depresión & 0.00 & $-0.34 *$ & -0.08 & -0.07 & -0.16 & $0.28 *$ \\
\hline Cólera & 0.00 & $-0.34 *$ & -0.04 & -0.15 & -0.20 & 0.22 \\
\hline Vigor & 0.00 & $-0.29 *$ & $-0.39 *$ & -0.12 & $-0.36 *$ & $0.46 *$ \\
\hline Fatiga & 0.02 & $-0.43 *$ & 0.08 & -0.21 & -0.24 & $0.27 *$ \\
\hline Confusión & 0.00 & $-0.33 *$ & -0.11 & -0.04 & -0.20 & $0.38 *$ \\
\hline Amistad & 0.21 & $-0.29 *$ & -0.16 & $-0.32 *$ & $-0.41 *$ & $0.49 *$ \\
\hline Escala & 0.00 & $0.34 *$ & -0.01 & 0.09 & 0.17 & -0.24 \\
\hline \multicolumn{7}{|c|}{ (c) Control } \\
\hline \multicolumn{7}{|c|}{ Rho Spearman } \\
\hline Variable & Shapiro & PIED & STAI & CSAI_C & CSAI_S & CSAI_A \\
\hline Tensión & 0.57 & $-0.50 *$ & 0.03 & $-0.27 *$ & $-0.49 *$ & $0.45 *$ \\
\hline Depresión & 0.00 & $-0.41 *$ & -0.09 & -0.24 & $-0.37 *$ & $0.46 *$ \\
\hline Cólera & 0.02 & $-0.34 *$ & -0.05 & $-0.29 *$ & $-0.38 *$ & $0.41 *$ \\
\hline Vigor & 0.07 & -0.21 & -0.06 & -0.13 & $-0.38 *$ & $0.46 *$ \\
\hline Fatiga & 0.03 & $-0.35 *$ & 0.07 & -0.20 & -0.25 & $0.28 *$ \\
\hline Confusión & 0.00 & $-0.50 *$ & -0.08 & -0.22 & $-0.40 *$ & $0.51 *$ \\
\hline Amistad & 0.40 & -0.24 & -0.05 & $-0.31 *$ & $-0.37 *$ & $0.43 *$ \\
\hline Escala & 0.03 & $0.43 *$ & 0.04 & 0.23 & $0.35 *$ & $-0.40 *$ \\
\hline
\end{tabular}

Si se analiza la escala Intensidad, se aprecian correlaciones medias y altas de carácter positivo entre el factor Tensión y la escala PIED $(r h o=.50 ; p<.05)$, Tensión y CSAI_somático $(r h o=.53 ; p<.05)$, entre el factor Depresión y la escala PIED $\left(r h o=.59 ; p^{<.05}\right)$, Depresión y CSAlautoconfianza (rho $=.53 ; p<.05)$, entre el factor $\mathrm{Vi}$ gor y CSAlautoconfianza $(r h o=.50 ; p<.05)$. Asimismo, se aprecian correlaciones medias y directas $(r h o=.61$; $p<.05$ ), entre el factor Fatiga y la escala PIED ( $r r_{0}=.62$; 
p .05). Al comparar la escala Intensidad del POMS-VIC con las escalas propuestas, se observan correlaciones medias y altas, de carácter negativo entre POMS_Intensidad y PIED (rho=-.61; $<<.05)$, y entre POMS_Intensidad y CSAIsomático (rho $=-.55 ; p<.05)$.

Si se atiende a la escala Valencia, se aprecian correlaciones medias e inversas, entre el factor Tensión y la escala PIED $(r h o=-.44 ; p<.05)$, y Fatiga y la escala PIED $(r h o=-.43 ; p<.05)$. Así como se aprecian correlaciones medias y positivas entre el factor Vigor y CSAIautoconfianza $(r h o=.46 ; p<.05)$, y entre Amistad y CSAlautoconfianza $(r h o=.49 ; p<.05)$.

Por último, al prestar atención a la escala Control, se aprecian correlaciones medias y altas, de carácter negativo, entre el factor Tensión y la escala PIED ( $r h o=-.50$; $p<.05)$, y entre Confusión y PIED (rho=-.50; $p<.05)$, así como correlaciones medias y positivas entre el factor Confusión y CSAlautoconfianza $(r h o=.51 ; p<.05)$.

\section{Discusión}

Este trabajo se ha centrado en presentar un estudio de validez convergente sobre una nueva versión del POMS que permite profundizar en el papel que tiene el estado de ánimo de los deportistas, partiendo de la propuesta de dos nuevas escalas a tener en cuenta en la valoración del estado de ánimo: Valencia y Control, y comparando los resultados obtenidos con otras escalas similares (PIED, STAI y CSAI-2R). Asimismo, y para simplificación de la escala POMS-VIC, se ha administrado a los participantes la versión ordenada (Borges et al., 2017) que simplifica la cumplimentación de la misma.

En este sentido, los resultados muestran cómo existen diferencias en función de las variable género y edad. A pesar de que el número de diferencias significativas no es elevado (especialmente en la variable edad), este dato puede deberse a la utilización de una muestra relativamente reducida. La segmentación de la muestra en función de la edad (mayores y menores de 25 años) puede constituirse como una división de grupos excesivamente amplia y gruesa, recomendándose en posteriores estudios incrementar la muestra con el objetivo de que existan rangos de edad más reducidos con un mayor número muestral en cada rango. A pesar de ello, el estudio muestra la sensibilidad del instrumento al establecerse algunas diferencias en función de las variables personales consideradas y los niveles de fiabilidad obtenidos, al igual que los valores obtenidos en estudios previos con la versión clásica del POMS lo que confirma la estructura teórica planteada.
Por otra parte, el análisis de las correlaciones obtenidas en las tres escalas permite inferir, desde una perspectiva de la validez convergente y discriminante de los factores, dos grupos de estados anímicos: un grupo hace referencia a Tensión, Depresión, Cólera, Fatiga y Confusión; y el otro grupo a Vigor y Amistad. Esta diferenciación es refrendada por las propias puntuaciones directas de las que informa el POMS-VIC, pues los deportistas declararon altos valores en Vigor y Amistad y bajos valores en el resto de estados. Estos resultados son congruentes con la escala Valencia, donde los valores de mayor agrado se situaron en Vigor y Amistad. En cualquier caso, cabe destacar que los deportistas manifestaron alto control de todos los estados emocionales.

En última instancia, al comparar los resultados obtenidos en el POMS-VIC y los obtenidos en el resto de cuestionarios (PIED, STAI y CSAI-2R), se encuentra que existe una relación considerable entre los diferentes instrumentos. Este resultado implica que la propuesta es válida para la medida del estado emocional y se justifica su uso en el ámbito deportivo por su facilidad de comprensión y aplicación.

Por tanto, el empleo del POMS-VIC se justifica por las aplicaciones prácticas que tiene la inclusión de las dimensiones Valencia y Control, que aquí se presentan y validan para configurar un perfil emocional completo del deportista, así como conocer con precisión la toma de conciencia que tiene el deportista sobre su propio estado emocional. La recogida de información sobre esa toma de conciencia del propio deportista puede mejorar los procesos de intervención y entrenamiento con deportistas una vez conocida la vivencia idiosincrática experimentada por el deportista respecto al estado emocional que percibe. Por otro lado, nos permite relacionar los estados de ánimo con la obtención de un rendimiento deportivo óptimo (De la Vega et al., 2013). A pesar de ello, es necesario profundizar entre las relaciones que se establecen para configurar el estado de ánimo del deportista más allá del empleo de la escala Intensidad, que es la que ha recibido la atención prioritaria hasta la fecha.

Una de las limitaciones encontradas en este trabajo, se relacionan con la falta de consenso en la definición de estado de ánimo, la variedad de interpretaciones y clasificaciones, lo que limita la posibilidad de realizar comparaciones más detalladas. Por otro lado, y dado que la recogida de datos se realizó mediante autoinforme, lo cual puede llegar a ofrecer medidas inexactas y engañosas al recurrir a la memoria del participante, quién debido a la falta de conocimiento, ganas o interés, puede 
ofrecer diferentes respuestas. En segundo lugar es importante destacar que se ha medido el estado emocional a través de la valoración de la intensidad de los sentimientos (más efímeros y volátiles) sin tener en cuenta la valoración y el control que ejerce sobre los mismos, el participante, resultando complicado establecer comparaciones y considerar el papel que juegan las diferencias individuales en la inducción del estado de ánimo. Por otro lado, se ha demostrado que las diferencias individuales influyen en el rendimiento (Morgan et al., 1988) y en el estado de ánimo precompetitivo (Prapavessis \& Grove, 1994), considerando que la personalidad podría inflar falsamente las relaciones entre estado de ánimo y rendimiento (Cerin et al., 2000).

Desde esta perspectiva, se tiene constancia de que un determinado estado de ánimo precompetitivo puede ser beneficioso o perjudicial para el rendimiento deportivo como es el caso de Tensión, Cólera y Vigor, que acompañados de altos niveles de excitación fisiológica son perjudiciales para el rendimiento. Sin embargo, si esos mismos estados de ánimo se acompañan de niveles moderados de activación fisiológica, serían beneficiosos, a través de la interrupción o aumento de la atención, el procesamiento de la información y la toma de decisiones (Brandt, Silveira, Brusque \& Andrade, 2016).

Por último, se considera que este estudio posee fortalezas que merecen resaltarse. Por una parte, el empleo de un instrumento de evaluación adaptado al contexto deportivo, lo cual abre nuevas vías y líneas de investigación, tanto en el campo deportivo como en otros campos afines, como el de la intervención psicológica. Cabe destacar que los hallazgos y resultados encontrados en este trabajo proporcionan información relevante al psicólogo deportivo, pero también al entrenador, profesor y educador deportivo; para comprender el amplio y complejo fenómeno de la percepción del estado emocional en el rendimiento deportivo, profesional, en el ámbito de la salud y el académico. No obstante, estos resultados proponen nuevos objetivos de investigación, como puede ser la influencia del estado emocional en la percepción de riesgo y la toma de decisiones.

Asimismo, futuros estudios deben profundizar en una serie de líneas prioritarias: en lo que respecta a los análisis psicométricos, resultan necesarios nuevos estudios ampliando la muestra a diferentes deportistas (nivel, edad y experiencia) y especialidades deportivas. De forma que se pudiera llegar a hablar de un perfil emocional específico que se relacione con una mejora del rendimiento para cada deporte y deportista, puesto que la activación ideal no será la misma para deportes de contacto como el rugby o deportes de precisión como el tiro con arco. Asimismo, resultaría interesante considerar por otro lado, además del estado emocional, otras variables de personalidad moduladoras del rendimiento deportivo como el optimismo, el estrés, la ansiedad y las expectativas de éxito (Ortín et al., 2011).

Estos aspectos, que suponen en parte algunas de las limitaciones del estudio, no menoscaban la potencialidad del POMS-VIC, considerando que puede ser uno de los instrumentos que más se empleen en el ámbito de la investigación básica y aplicada de las relaciones establecidas entre el estado de ánimo y el rendimiento deportivo.

Asimismo, resulta necesario, una vez comprobada su validez en la medida del estado de ánimo y su congruencia, el estudio experimental de su uso como variable predictora del rendimiento deportivo en situaciones de competición real o similar.

\section{Conclusiones}

En base a los resultados aquí expuestos podemos confirmar que este estudio demuestra la validez de constructo de la prueba al comparar los resultados obtenidos en el POMS-VIC y los obtenidos en otras pruebas similares (PIED, STAI y CSAI-2R). Esto prueba que existe una relación considerable entre ambos instrumentos. A su vez, esta propuesta es válida para la medida del estado emocional y se justifica su uso en el ámbito deportivo, en tanto facilitan los procesos de intervención con deportistas por parte del psicólogo deportivo y ofrecen a entrenadores y responsables deportivos información útil para mejorar el proceso de intervención en el entrenamiento y competición.

\section{Referencias}

Abenza, L., González, J., Reyes, L., Reyes, F., Blas, A., \& Olmedilla, A. (2014). Descripción y evaluación del entrenamiento psicológico de una deportista de regata de clase láser radial. Revista Iberoamericana de Psicología del Ejercicio y el Deporte, 9(1), 67-92.

Andrade, E., Arce, C., \& Seoane, G. (2000). Aportaciones del POMS a la medida del estado de ánimo de los deportistas: estado de la cuestión. Revista de Psicología del Deporte, 9(12), 8-20.

Andrade, E., Lois, G., \& Arce, C. (2007). Propiedades psicométricas de la versión española del inventario de ansiedad competitiva CSAI-2R en deportistas. Psicothema, 19(1), 150-155. 
Arroyo, R., González, O., \& Arruza, J. A. (2020). Influencia del sexo y el resultado en el estado de ánimo de futbolistas iniciados. Retos, 38, 349-354. https: / /doi.org/10.47197/ retos.v38i38.74248.

Balaguer, I., Fuentes, I., Meliá, J. L., García-Merita, M., \& Pérez, G. (1993). El perfil de los estados de ánimo (POMS): Baremo para estudiantes valencianos y su aplicación en el contexto deportivo. Revista de Psicología del deporte, 2(2), 39-52.

Bar-On, R., \& Parker, J. (2000). The handbook of emotional intelligence: theory, development, assessment, and application at home, school, and in the workplace. San Francisco: JosseyBass.

Barrios, R., \& González, L. (2012). Elaboración de un instrumento para evaluar estados de ánimo en deportistas de alto rendimiento (M. Fajardo, Ed.). Universidad de Ciencias de la Cultura Física y el Deporte.

Ben-Zur, H., \& Yagil, D. (2005). The relationship between empowerment, aggressive behaviours of customers, coping, and burnout. European Journal of Work and Organizational Psychology, 14 (1), 81-99. dx.doi.org/ $10.1080 / 13594320444000281$

Borges, P. J., Ruiz-Barquín, R., \& De laVega Marcos, R. (2017). Análisis y validación psicométrica de una nueva forma de presentación (ordenada) del Perfil de Estados de Ánimo (Valencia, Intensidad, Control) POMS-VIC. Retos, 32(2), 82-87.

Brandt, R., Silveira, M., Brusque, T., \& Andrade, A. (2016). Asociación entre estado de ánimo y el rendimiento de regatistas brasileños de élite: Ganadores vs. no ganadores. Cultura, ciencia y deporte, 11(32), 119-125. dx.doi.org/ 10.12800/ccd.v11i32.712

Cerin, E., Szabo, A., Hunt, N., \&Williams, C. (2000). Temporal patterning of competitive emotions: A critical review. Journal of Sports Sciences, 18(8), 605- 626. doi.org/ 10.1080/02640410050082314

De laVega, R., Galán,A., Ruiz, R., \&Tejero, C. (2013). Estado de ánimo precompetitivo y rendimiento percibido en Boccia Paralímpica. Revista de Psicología del Deporte, 22(1), $39-45$.

De laVega-Marcos, R., Ruiz-Barquín, R., Borges, P., \& TejeroGonzález, C. Ma , (2014). Una nueva medida tridimensional del estado de ánimo deportivo: el POMSVIC. Cuadernos de Psicología del Deporte, 14(2), 37-46.

Fierro-Suero, S., Velázquez-Ahumada, N., \& FernándezEspínola, C. (2021). La influencia del clima de aula sobre las emociones del alumnado. Retos, 42, 432-442. https: / /doi.org/10.47197/retos.v42i0.87353

Guillén-Riquelme, A., \& Buela-Casal, G. (2011). Actualización psicométrica y funcionamien-to diferencial de los ítems en el State TraitAnxiety Inventory (STAI). Psicothema, 23(3), 510-515.

Hanin, Y. (2007). Emotions in sport: current issues and perspectives. En Handbook of sport psychology. New Jersey:
John Wiley \& Sons.

Lang, P. J. (2010). Emotion and motivation: Towards consensus definitions and a common research purpose. Emotion Review, 2(3), 229-233. dx.doi.org/10.1177/ 1754073910361984

Martens, R.,Vealey, R., \& Burton, D. (1990). Competitive anxiety in sport. Champaign, IL: Human Kinetics.

McNair, D. M., Lorr, M., \& Droppelmann, L. F. (1971). Manual for the Profile of Mood States. San Diego: CA: Educational and Industrial.

Molano-Tobar, N. J., Rojas, E. A., \&Vélez, R. A. (2021). Estado emocional y su correlación con las habilidades de afrontamiento de universitarios, Popayán-Colombia. Retos, 42, 154-161. https: / / doi.org/10.47197/ retos.v42i0.86359.

Montero, I., \& León, O. (2007). A guide for naming research studies in psychology. International. Journal of Clinical Health \& Psychology, 7(3), 847-862.

Morgan, W. (1980). Test of Champions: the iceberg profile. Psychology. Today, 14, 92-99.

Morgan,W., O’Connor, P., Ellickson, K., \& Bradley, P. (1988). Personality structure, mood states, and performance in elite male distance runners. International Journal of Sport Psychology, 19(4), 247-263.

Ortín, F., Garcés de los Fayos, E., Gosálvez, J., Ortega, E., \& Olmedilla. A. (2011). Optimismo y ejecución en el deporte en situaciones adversas. Replicando a Seligman 1990. Revista de Psicología del Deporte, 20(2), 491-501.

Prapavessis, H. (2000). The POMS and sports performance: A review. Journal of Applied Sport Psychology, 12(1), 34-48. dx.doi.org/10.1080/10413200008404212

Prapavessis, H., \& Grove, R. (1994). Personality variables as antecedents of precompetitive mood state. International Journal of Sport Psychology, 25(1), 85-99.

Puig, N. (2012). Emociones en el deporte y sociología. RICYDE. Revista Internacional de Ciencias del Deporte, 8(28), 106-108. doi:10.5232/ricyde2012.028.

Ruiz-Barquín, R. R. (2005). Análisis de las diferencias de personalidad en el deporte del judo a nivel competitivo en función de la variable sexo y categoría de edad deportiva. Cuadernos de psicología del deporte, 5 (1-2), 28 48.

Salkind, N. J. (1999). Métodos de investigación. México: Prentice Hall.

Spielberger, C., Gorsuch, R., \& Lushene, R. (1970). Manual for the State-Trait Anxiety Inventory. California: Consulting Psychologist Press.

Torres-Luque, G., Hernández-García, R., Ortega, E., \& Olmedilla, A. (2010). Estudio de casos sobre el perfil de los estados de ánimo en judokas a lo largo de un período competitivo. Revista Iberoamericana de Psicología del Ejercicio y el Deporte, 5(1), 89-98. 